\title{
From the biology of PP2A to the PADs for therapy of hematologic malignancies
}

\section{Maria Ciccone $^{1}{ }^{*}$, George A. Calin ${ }^{1}$ and Danilo Perrotti ${ }^{2}$}

${ }^{1}$ Department of Experimental Therapeutics, MD Anderson Cancer Center, The University of Texas, Houston, TX, USA

${ }^{2}$ Department of Medicine, The Greenebaum Cancer Center, University of Maryland School of Medicine, Baltimore, MD, USA

\section{Edited by:}

Barbara Pro, Fox Chase Cancer

Center, USA

Reviewed by:

Annie Im, University of Pittsburgh

Cancer Institute, USA

Deepa Sampath, Ohio State

University, USA

*Correspondence:

Maria Ciccone, Department of

Leukemia, The University of Texas MD

Anderson Cancer Center, 1515

Holcombe Blvd, Houston, TX 77030,

USA

e-mail:cccmra@unife.it

Over the past decades, an emerging role of phosphatases in the pathogenesis of hematologic malignancies and solid tumors has been established. The tumor-suppressor protein phosphatase 2A (PP2A) belongs to the serine-threonine phosphatases family and accounts for the majority of serine-threonine phosphatase activity in eukaryotic cells. Numerous studies have shown that inhibition of PP2A expression and/or function may contribute to leukemogenesis in several hematological malignancies. Likewise, overexpression or aberrant expression of physiologic PP2A inhibitory molecules (e.g., SET and its associated SETBP1 and CIP2A) may turn off PP2A function and participate to leukemic progression. The discovery of PP2A as tumor suppressor has prompted the evaluation of the safety and the efficacy of new compounds, which can restore PP2A activity in leukemic cells. Although further studies are needed to better understand how PP2A acts in the intricate phosphatases/kinases cancer network, the results reviewed herein strongly support the development on new PP2A-activating drugs and the immediate introduction of those available into clinical protocols for leukemia patients refractory or resistant to current available therapies.

\section{Keywords: PP2A, PADs, SET, phosphatases, tumor suppressor}

\section{INTRODUCTION}

The phosphorylation status of proteins is crucial in regulating their functions and properties. In normal cells, kinases and phosphatases participate to phosphorylation processes (1). By modulating the phosphorylated load, they control the on-off switch of target proteins. The balance between kinase and phosphatase activities is critical in many hematological malignancies. Although numerous studies highlighted the role of kinases in malignant transformation, much less is known about the contribution of specific phosphatases to cancer development.

Oncogenic kinases and tumor-suppressor phosphatases maintain the cell homeostasis by exerting their activities on cell growth, survival, and differentiation. Thus, aberrant oncogene expression and loss of tumor-suppressor gene function affect cell cycle, apoptosis, and DNA damage repair machineries. $A B L 1$ is a wellcharacterized kinase that has been involved in leukemic transformation (2). The break cluster region (BCR)-ABL1 oncoprotein is a tyrosine-kinase that constitutively activates downstream signaling thereby inducing cell proliferation, survival, and clonal expansion in chronic myeloid leukemia (CML) and a cohort of acute lymphoblastic leukemia (ALL) patients. In addition to the tumorsuppressors TP53, RB1, and ATM, usually lost in several leukemias, inhibition of specific phosphatases expression or loss of their function has been recently detected in many cancers (3). Interestingly, there is increasing evidence that unrestrained oncogene kinase activity is not sufficient to transform cells if a concurrent inhibition of the antagonizing tumor-suppressor phosphatases does not occur (4).

The introduction of tyrosine-kinase inhibitors [TKIs; e.g., imatinib (IM)] has dramatically changed the clinical outcome of patients with hematological malignancies (5). However, the occurrence of mutations at the active kinase site or additional chromosomal/molecular abnormalities resulting in drug resistance and, possibly, disease progression (6) indicate that new strategies are needed to prolong survival of leukemia patients resistant or refractory to current chemo and TKI-based therapies. Although the rescue of tumor-suppressor function seems to be an intriguing challenge in cancer treatment, few molecules have been proven to target specifically tumor-suppressors.

Protein phosphatase 2A (PP2A) refers to a family of serinethreonine phosphatases that accounts for the majority of serinethreonine phosphatase activity in eukaryotic cells (7). In the last decade, an emerging role of PP2A in the onset and progression of solid tumors and hematological malignancies has been established $(8,9)$. These findings led to the discovery and development of a new family of compounds, the PP2A-activating drugs (PADs) that are capable of restoring PP2A phosphatases activity of most of them through interference between PP2A and its inhibitor SET/I2PP2A $(8,10)$. Pre-clinical studies proved the efficacy and no-toxicity of PADs in several ex vivo and in animal studies (8), indicating that PP2A reactivation in combination with kinase inhibition or chemotherapy represents a feasible and effective strategy for the treatment of hematological malignancies.

\section{PP2A IN NORMAL CELLS \\ PP2A STRUCTURE AND REGULATION}

The PP2A core enzyme consists of a $36 \mathrm{kDa}$ catalytic $\mathrm{C}$ subunit $\left(\mathrm{PP} 2 \mathrm{~A}_{\mathrm{C}}\right)$ and a $65 \mathrm{kDa}$ structural A subunit $\left(\mathrm{PP} \mathrm{A}_{\mathrm{A}}\right)$. In mammals, two isoforms ( $\alpha$ and $\beta$ ) are encoded for each subunit $(A \alpha, A \beta, C \alpha$, and $C \beta)$ (11). The structure of the PP2A complex is 
enriched with a regulatory B subunit. Four families of the B subunit, with a molecular weight ranging from 50 to $130 \mathrm{kDa}$, have been identified: PP2A $\mathrm{A}_{\mathrm{B}}$ (B55 or PR55), PP2A $\mathrm{A}_{\mathrm{B}^{\prime}}$ (B56 or PR61), PP2A $_{\mathrm{B}^{\prime \prime}}$ (B72 or PR72; B130 or PR130; PR48; and G5PR) PP2A (PR93 and PR110). By assembling differently the A, B, and C subunit isoforms, more than 75 distinct PP2A holoenzymes could be built up (12).

The $\mathrm{A}$ and $\mathrm{C}$ subunits are evolutionary conserved and ubiquitously expressed. These two subunits form a catalytic complex $\left(\mathrm{PP} 2 \mathrm{~A} / \mathrm{A}_{\mathrm{C}}\right)$ that can interact with the regulatory $\mathrm{B}$ subunits or certain viral antigens (e.g., polyoma small $\mathrm{T}$ and middle $\mathrm{T}$ antigens, and SV40 small tumor antigen) to affect activity and determine PP2A substrate and tissue specificity $(12,13)$. Moreover, the $\mathrm{B}$ subunit may vary depending on stage-development (14, $15)$ and it can recruit $\mathrm{PPA} 2 / \mathrm{A}_{\mathrm{C}}$ to a selective subcellular compartment (cytoplasm or nucleus) driving it to a specific substrate target (1).

The ability of PP2A to regulate different cellular activities depending on its structure and composition renders this enzyme a suitable target for new therapeutic compounds as improving the drug affinity for each isoform might entail high tissue specificity and better safety profile.

\section{PP2A PATHWAYS}

Protein phosphatase $2 \mathrm{~A}$ is involved in a consistent number of physiological processes in adult and embryonic cells (Figure 1). For example, the transcription factor $\beta$-catenin, which is the major effector in the Wnt signaling pathway both in cancer and embryonic development $(12,16)$ undergoes PP2A-dependent regulation. $\mathrm{PP} 2 \mathrm{~A}$ also plays an essential role in cell division and apoptosis depending on the specific isoform constituting the PP2A holoenzyme $(17,18)$. Indeed, PP2A is required to maintain G1/S cyclin levels by modulating their phosphorylation status, a necessary event to properly transit through the cell cycle (19).

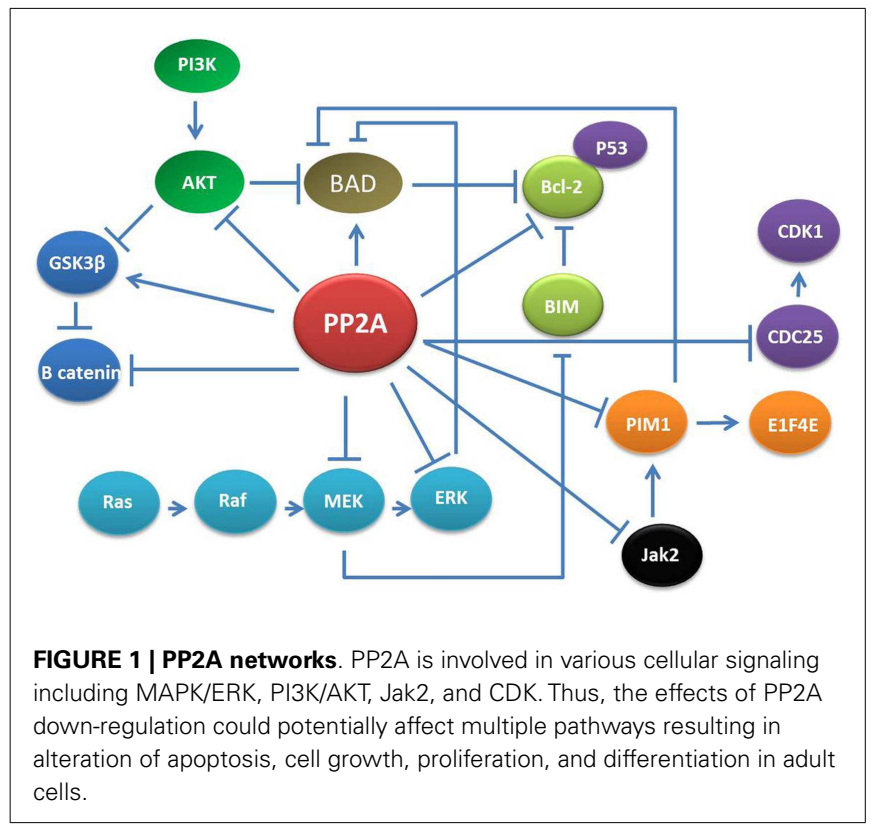

Notably, PP2A loss-of-function results in inhibition of apoptosis. This mostly depends on interference with two major mitogenic/survival pathways: the MAPK/ERK and the PI3K/AKT cascades. In normal cells, specific PP2A complexes desphosphorylate and inactivate MEK and ERK1 kinases thereby keeping the activity of many signal transducers, apoptosis regulators, and transcription factors (e.g., STAT5 and 3; c-Myc) tightly regulated. PIM1 kinase protein is a target of PP2A and once it is dephosphorylated by PP2A, it is degraded by the proteasome machinery (20). Furthermore, PIM1 represses the pro-apoptotic molecule BAD through its phosphorylation at Ser112 gatekeeper site (21). Likewise, PP2A suppresses PI3K/AKT-generated signals mostly by direct AKT dephosphorylation but also through activation of GSK-3b or inhibition of cytokine-generated signals, which lead to Akt-activation $(22,23)$.

In addition to the MAPK/ERK and PI3K/AKT cascades, PP2A function is strictly related with the Janus kinase 2 (Jak2) tyrosine kinase $(24,25)$. In IL-3-stimulated myeloid progenitor cells, the interaction between Jak2 and PP2A transiently increases and Jak2 becomes activated and PP2Ac activity suppressed by phosphorylation on Y307 (26). In primary myeloid stem and progenitors cells, reactivation of $\mathrm{PP} 2 \mathrm{~A}$ also induces Jak2 inactivation thus resulting in shut-down of mitogenic/survival cytokine-generated signals (27).

\section{PP2A INHIBITORS}

Physiologic PP2A inhibitors seem to be involved in the mechanisms of progression and aggressiveness of hematological malignancies. ANP32A (I1PP2A), SET (I2PP2A), CIP2A, SETBP1A, type 2A-interacting protein (TIP), and ENSA are well-characterized cellular PP2A inhibitory molecules (Table 1).

Table 1 | PP2A inhibitors in hematological malignancies

\begin{tabular}{lll}
\hline PP2A inhibitors & Pattern of expression & $\begin{array}{l}\text { Hematological } \\
\text { malignancies }\end{array}$ \\
\hline SET (I2PP2A) & $\begin{array}{l}\text { Overexpression/gain of } \\
\text { function }\end{array}$ & $\begin{array}{l}\mathrm{CML}, \mathrm{CLL}, \mathrm{NHL} \text { cell line, } \\
\text { Jak2 }{ }^{\mathrm{V} 617} \mathrm{MPN}, \mathrm{MML}\end{array}$ \\
SETBP1 & $\begin{array}{l}\text { Mutations } \\
\text { Overexpression/gain of } \\
\text { function }\end{array}$ & $\begin{array}{l}\mathrm{aCML}, \mathrm{SAML}, \mathrm{CMML}, \mathrm{CNL}, \\
\text { unclassified MDS/MPN }\end{array}$ \\
& &
\end{tabular}

CIP2A

Overexpression

CML

ANP32A (I1PP2A) Overexpression

K562 cell line

TIP

Unknown

Unknown

ENSA

Unknown

Unknown

CML, chronic myeloid leukemia; CLL, chronic lymphocytic leukemia; NHL, non-

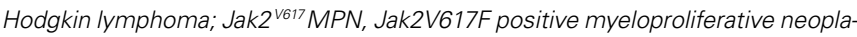
sia; $A M L$, acute myeloid leukemia; aCML, atypical chronic myeloid leukemia; SAML, secondary acute myeloid leukemia; CMML, chronic myelomonocytic leukemia; CNL, chronic neutrophilic leukemia; unclassified MDS/MPN, unclassified myelodisplastic/myeloproliferative neoplasia. 
SET and SET binding protein (SETBP1) act synergistically and permit the formation of the SETBP1/SET/PP2A complex, which inhibits PP2A phosphatase activity (28). SET (or I2PP2A) is a potent endogenous inhibitor of the tumor-suppressor PP2A (8) as PP2A acts as a negative regulator of several survival and proliferation pathways that are aberrantly activated in hematological malignancies (8). In addition, it has been reported that SET inhibits the DNase activity of the tumor-suppressor NM23-H1 and that the cleavage of SET by Granzyme A during the cytotoxic T lymphocyte-induced apoptosis releases NM23-H1 from inhibition and triggers NM23-H1 to translocate into the nucleus, where it cleaves the DNA $(29,30)$.

\section{SETBP1}

SETBP1 localizes on chromosome 18q21.1 and codes for a nuclear protein of $170-\mathrm{kDa}$ that specifically interacts with SET forming a heterodimer (31). SETBP1 germline mutations have been described in Schinzel-Giedion syndrome, a rare congenital disorder characterized by aberrant bone formation (32) and predisposition to myeloid malignancies. SETBP1 overexpression protects SET from protease cleavage, increasing the amount of full-length SET protein and leading to the formation of the SETBP1-SETPP2A complex that results in PP2A inhibition (28). Interestingly, not only SETBP1 overexpression but also SETBP1 mutations have been associated with hematological disorders $(28,33,34)$. Cells expressing SETBP1 Gly870Ser variant showed higher level of SETBP1 and SET proteins, and significantly reduced PP2A activity indicating that SETBP1 mutations contribute to SET stabilization and PP2A inhibition (34).

An additional PP2A inhibitor is the cancerous inhibitor of $P P 2 A$, e.g., CIP2A, which normally prevents PP2A-mediated dephosphorylation of $\mathrm{c}-\mathrm{Myc}$ at serine $62 . \mathrm{pS}^{62}-\mathrm{Myc}$ is more stable than its dephosphorylated counterpart, and CIP2A therefore prevents c-Myc degradation (35). Moreover, impaired p53 activity was demonstrated to increase CIP2A expression trough E2F1, which in turn, by inhibiting PP2A activity, increases stabilizing E2F1 (36).

Whereas in vivo data have confirmed the involvement of SET, SETBP1, and CIP2A proteins in leukemic transformation process, so far we could not rule out the contribution to the development of hematological malignancies of other molecules that have been proven to inhibit PP2A including TIP, ANP32A, and ENSA. Recently, one study has reported that knock-down of the NM23$\mathrm{H} 1$ factor in CML K562 cell line decreased proliferation and altered the expression of ANP32A, a potent and selective PP2A inhibitor (37).

Although variations exist between increased levels of PP2A inhibitors and adverse clinical outcome in different hematologic malignancies, pharmacologic suppression of PP2A inhibitors represents an effective strategy to induce apoptosis of leukemic cells and/or sensitize resistant cells to TKIs $(25,27,38-40)$. Thus, PP2A reactivation should be taken as a possible therapeutic approach.

\section{PP2A AND HEMATOLOGICAL MALIGNANCIES CHRONIC MYELOID LEUKEMIA AND MYELOPROLIFERATIVE DISORDERS Chronic myeloid leukemia}

Chronic myeloid leukemia is a myeloproliferative disorder arising from the transformation of the hematopoietic stem cell (HSC) and characterized by exuberant cell expansion and altered differentiation of myeloid precursors. The molecular hallmark of CML is the Philadelphia chromosome $(\mathrm{Ph})$ that derives from the translocation $\mathrm{t}(9 ; 22)$ and causes the juxtaposition of the $A B L 1$ kinase gene with the break cluster region gene $(B C R)(2)$. The BCR-ABL1 fusion oncoprotein is a constitutively active tyrosine kinase that confers growth factor-independent proliferation and enhanced survival to the hematopoietic progenitors harboring this abnormality. Although TKIs control the disease in vast majority of patients, a small proportion of patients with CML will eventually lose response to TKIs treatment and progress to blast crisis (BC) (41). The occurrence of mutations at the active site of $B C R-A B L 1$ may at least in part explain the overcoming resistance in patients treated with TKIs (42). Moreover, the persistence of quiescent CML HSCs might contribute to disease progression because TKIs seem to not affect leukemic stem cell proliferation and survival (43). In CML cells, PP2A is a key target in BCR-ABL1 downstream signaling and, in fact, the BCR-ABL1 oncoprotein prevents PP2A auto-dephosphorylation at tyrosine $307(44,45)$ thereby keeping PP2A in its inactive status. By targeting $\mathrm{BCR} / \mathrm{ABL} 1$, TKIs inhibit ABL kinase activity and restore PP2A function. Interestingly, in IM-resistant CML cells, the sphingosine kinase-1 (SK-1)/sphingosine 1-phosphate (S1P) activation enhances BCR-ABL1 stability through S1P receptor 2 (S1P2) signaling, which prevents BCR-ABL1 dephosphorylation and degradation through inhibition of PP2A (45). Molecular and pharmacological interference with SK-1/S1P2 may restore PP2A-dependent dephosphorylation and enhance IM- or nilotinib-induced growth inhibition in primary $\mathrm{CD} 34^{+} \mathrm{CML}$ progenitors and $\mathrm{BCR}-\mathrm{ABL} 1+$ cell lines.

Recently, it has been shown that persistence of leukemic HSCs in bone marrow of patients with CML requires the inhibition of the PP2A and BCR-ABL1 expression but not its activity (27). In comparison with HSCs from healthy individuals, PP2A activity was remarkable suppressed in HSCs from patients with CML (27). This result is apparently in contrast with the low BCR-ABL1 tyrosinekinase activity found in the $\mathrm{Ph}+\mathrm{HSC}$-enriched cell fractions, which would have expected to be associated with PP2A restoration. However, BCR-ABL1 expression is needed for the recruitment and activation of Jak2 that, in turn, triggers SET-dependent PP2A inhibition and activation of $\beta$-catenin-mediated self-renewal/survival signals (27). The consistent difference in PP2A activity in normal and leukemic HSCs implies that restoration of PP2A activity would allow the selective targeting of leukemic HSCs without compromising the normal counterpart (Figure 2).

An additional finding is that in CML stem cells not only PP2A but also PP2A inhibitory molecules might be aberrantly expressed and might contribute to low PP2A activity. SET was the first PP2A inhibitory molecule to have been found up-regulated in $\mathrm{CML}(\mathrm{CP}$ and $\mathrm{BC})$ and $\mathrm{Ph}+\mathrm{B}-\mathrm{ALL} \mathrm{CD} 34+$ progenitors through a BCR-ABL1 dose- and kinase-dependent manner (38). In CD34+ primary cells from patients with CML-BC and in $\mathrm{BCR}-\mathrm{ABL} 1+$ cell lines, SET expression is enhanced by BCR/ABL and increases during CML disease progression. In fact, SET protein levels correlate with $\mathrm{BCR} / \mathrm{ABL}$ activity and are higher in CML-BC CD34+ than CML-CP CD34+ cells and in CML-CP $\mathrm{CD}^{+}{ }^{+}$cells than in CD34+ cells from healthy donors (44). 


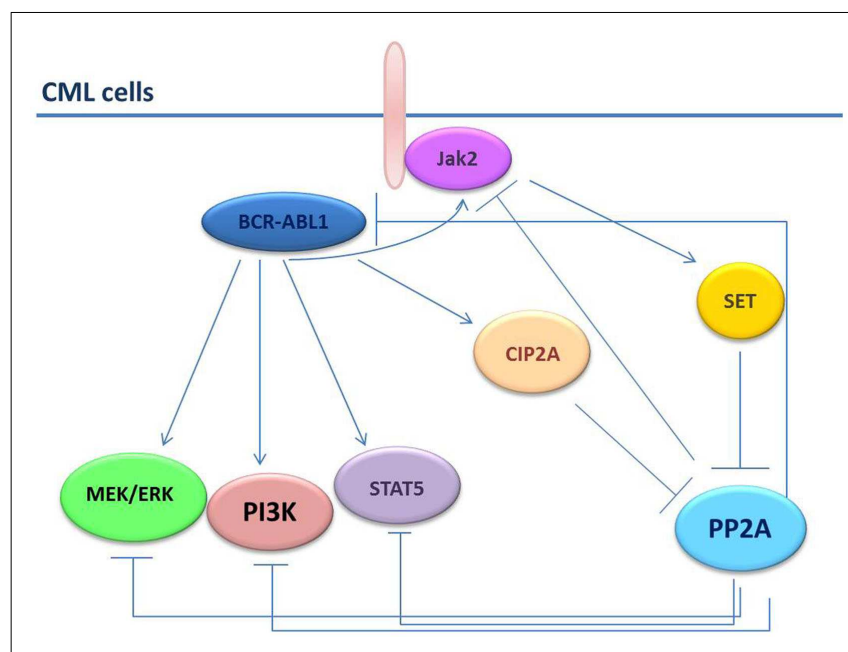

FIGURE 2 | PP2A in CML cells. In CML cells, the BCR-ABL1 and the PP2A pathways are strictly connected. PP2A induces dephosphorylation/inactivation of BCR-ABL1 and Jak2 tyrosine kinases. Conversely, BCR-ABL1 induces SET and CIP2A expression thus increasing the inhibition on PP2A. Jak2 also down-regulates PP2A through SET.

SET-mediated PP2A inactivation is essential for survival/selfrenewal of quiescent CML HSCs and maintenance of an active Jak2- $\beta$-catenin pathway and that PP2A is suppressed in CML HSCs in a BCR/ABL kinase-independent, Jak2-dependent manner (27). In BCR/ABL transformed cells, the levels of PP2A structural subunit PR65/A were found to correlate with increased levels of PP2Ac phosphorylation on tyrosine 307 which, as reported, inhibits PP2A activity (44). Accordingly, PP2ATyr307 levels are markedly reduced upon SET down regulation by means of shorthairpin RNA (shRNA) or IM treatment (44). The restoration of PP2A phosphatase activity induces the SHP-1-dependent BCRABL1 dephosphorylation/inactivation and triggers BCR-ABL1 proteasome degradation (44).

Likewise, CIP2A have been found overexpressed in hematological malignancies and in solid tumors, and to be associated with high proliferation rate and poor prognosis $(46,47)$. In a retrospective study, Lucas et al. have shown that high CIP2A protein levels are elevated at diagnosis in mononuclear cells (MNCs) and CD34+ cells from patients with CML who progressed into $\mathrm{BC}$ compared to patients who achieved a complete cytogenetic response (CCyR) or who had not progressed (48). Interestingly, in patients who progressed into CML-BC, the CIP2A levels were sustained despite IM treatment and low Sokal score. The inhibition of CIP2A resulted in a reduction of inactivated phosphorylated PP2ATyr307 fraction and BCR/ABL1 kinase activity. The knockdown of CIP2A led to a consistent drop of SET and Jak2 protein levels, suggesting the existence of an interplay between the PP2A complexes, their physiologic inhibitors (e.g., SET and CIP2A), and the BCR-ABL1/JAK2 signalosome (48).

Protein phosphatase $2 \mathrm{~A}$ is also inactivated in polycythemia vera (PV), essential thrombocytosis (ET), and primary myelofibrosis (PMF) and other myeloproliferative disorders (MPDs) characterized by the expression of the transforming Jak $2^{\mathrm{V} 617 \mathrm{~F}}$ oncogene
(25). Consistent with significant reduction of PP2A activity in $\mathrm{CD} 34+$ progenitors from patients with Jak2 ${ }^{\mathrm{V} 617 \mathrm{~F}}$ positive ET, PV, and MFI, treatment with Jak inhibitors may rescue PP2A activity through SET silencing (25).

Taken together, these data indicate that the balance among PP2A, SET, and CIP2A may play a crucial role not only for maintenance of CML progenitors both in chronic and blastic phase but also for the population of leukemic stem cells showing innate resistance to TKI. This observation has strong critical clinical implications as drugs that are able to rescue PP2A activity could potentially eradicate the leukemic HSCs reservoir or be effective in patients who progress to $\mathrm{BC}$ that so far define a clinical stage characterized by treatment refractoriness and poor prognosis.

\section{MYELODYSPLASTIC SYNDROME AND}

\section{MYELODYSPLASTIC/MYELOPROLIFERATIVE NEOPLASMS}

In other myeloproliferative malignancies, inhibition of PP2A is achieved by SET stabilization through its interaction with SETBP1 (31). Recently, by exome sequencing technology, SETBP1 mutations were found to recur frequently in patients with atypical chronic myeloid leukemia (aCML), a disorder with overlapping clinical features with CML but without the typical Ph1 translocation (34). Out of 644 patient samples with different myeloid and lymphoid malignancies and 344 cell hematopoietic and non-hematopoietic lines, the pGly870Ser SETBP1 mutation occurred in $24 \%$ of patients with aCML, and in 25,10 , and $4 \%$ of patients with chronic neutrophilic leukemia $(C N L)$, unclassified MDS/MPN disorders, and chronic myelomonocytic leukemia $(C M M L)$, respectively. Ectopic SETBP1 Gly870Ser expression in TF1 enhanced cell proliferation, which likely resulted from suppression of SETBP1 ubiquitination/degradation that, in turn, led to SET stabilization and PP2A inhibition (34). In primary aCML cells as well as in cells overexpressing the mutated SETBP1, SETBP1 levels correlated with increased expression of Lyn and PTGS2 (two known targets of PP2A) and induction of the TGF- $\beta$ pathway. Interestingly, aCML patients with SETBP1 mutations had a worse prognosis than those with wild-type SETBP1 (34).

Similar to what was found in aCML, a different group identified recurrent somatic mutations in SETBP1 by whole-exome sequencing of individuals with various myeloid malignancies (49). The mutations (pAsp868Asn, pGly870Ser, pIle871Thr, pSer869Asn, and pAsp880Asn or pAsp880Glu) in this cohort of patients corresponded with the recurrent de novo germline mutations responsible of Schinzel-Giedion syndrome and were detected in 15\% of CMML cases and $17 \%$ of secondary acute leukemia (sAML). Mutant cases were associated with advanced age, poor cytogenetic abnormalities, and shorter survival. However, in CMML subgroup, SETBP1 retained its prognostic value in association with $C B L$ mutations. Furthermore, the sequential analysis of serial samples at multiple time points of the disease course, in two different series of patients with myeloid malignancies, showed that SETBP1 mutations were absent at the time of initial presentation while increasing in allele frequencies by time $(49,50)$. These observations suggest that SETBP1 mutations are acquired during leukemic evolution contributing to leukemic progression rather than leukemic transformation. Transduction with mutant SETBP1 led to the immortalization of mouse myeloid precursors 
that acquired enhanced proliferative capacity and tyrosine phosphorylation of PP2A. In addition, a significantly higher HOXA9 and HOXA10 expression levels were observed in SETBP1-mutant leukemias in comparison with wild-type cases without SETBP1 overexpression (49). Overall, SETBP1 mutations predict poor outcome irrespective of age, sex, International Prognostic Scoring System (IPSS), and unfavorable cytogenetic risk $(49,50)$. However, the adverse prognostic value of SETBP1 mutations was not confirmed in two cohorts of patients with aCML and CMML, probably due to the fact that the median follow-up was relatively short (33).

Furthermore, SETBP1-mutant cases have been associated with higher white blood cell counts, high grade thrombocytopenia, and anemia than wild-type cases (33), with poor risk cytogenetic abnormalities like monosomy $7 /$ del7q $(33,49,50)$ and isochromosome $\mathrm{i}(17)(33,50)$. Interestingly, in patients with $\mathrm{i}(17)(\mathrm{q} 10)$, the TP53 and SETBP1 mutations are mutually exclusive; likewise, SETBP1 mutations rarely occur in cases harboring Jak2 ${ }^{\mathrm{V} 617 \mathrm{~F}}, \mathrm{MPL}^{\mathrm{W} 515}$, Jak2 exon 12, and TET2 mutations (33). Conversely, ASXL1 and CBL mutations correlate significantly with the occurrence of SETBP1 mutations $(33,34,49,50)$.

Although further are warranted, SETBP1 mutations seem to define a subgroup of patients with myeloid disorders characterized by specific clinical features and worse prognosis.

\section{ACUTE MYELOID LEUKEMIA}

An increasing number of studies have shown that the PP2A pathway is also affected not only in CML but also in other myeloid malignancies. Over the past few years, large-scale genomic studies have unveiled the presence of novel genetic aberrations in patients with acute myeloid leukemia (AML). C-KIT is a type-3 tyrosinekinase receptor activating multiple proliferation, differentiation, and survival signals in stem cells (51). Activating c-KIT mutations have been differently associated with higher relapse rate, shorter survival, and TKIs resistance (52). Interestingly, PP2A structural and regulatory subunits were found significantly reduced in myeloid cells expressing activated c-KIT mutants, meaning that PP2A is a crucial mediator of c-KIT leukemogenesis (40). Accordingly, the overexpression of PP2A-A $\alpha$ in D816V c-KIT cells induced apoptosis and inhibited proliferation (40), suggesting that the restoration of $\mathrm{PP} 2 \mathrm{~A}$ activity could represent an effective strategy to overcome drug resistance in $\mathrm{c}-\mathrm{KIT}^{+} \mathrm{AML}$.

Cristobal et al. described a novel translocation $\mathrm{t}(12 ; 18)(\mathrm{p} 13 ; \mathrm{q} 12)$ involving ETV6 in a patient with AML. The translocation resulted in overexpression of SETBP1 (18q12), located close to the breakpoint. They demonstrated that in cell lines transfected with a plasmid expressing SETBP1, its ectopic expression leads to increased full-length SET protein levels. In fact, the overexpression of SETBP1 might protect SET from protease cleavage and promotes the formation of a SETBP1/SET/PP2A complex that results in $\mathrm{PP} 2 \mathrm{~A}$ inhibition and increased proliferation rate of the leukemic cells (28). By quantitative RT-PCR, they observed that overexpression of SETBP1 occurred in $27.6 \%$ of patients with AML and was associated with unfavorable cytogenetic prognostic group, monosomy 7, and EVI1 overexpression. Similarly to patients with SETBP1 mutations, overexpression of SETBP1 in AML has also been associated with significantly shorter overall survival especially in elderly population (28). Accordingly, increased $\mathrm{pY}^{307}$ PP2A levels were observed in $78.4 \%$ of a cohort of patients that included de novo and secondary AML (53). In approximately twothird of patients, the PP2A inhibition was attributable to SET and/or SETBP1 overexpression. However, $34 \%$ of the patients with inactivated PP2A had either FLT3 or NPM1 aberrations, suggesting that PP2A is a secondary rather than a primary event in the process of leukemogenesis (53). Recently, SET expression studies in AML cell lines and CD34+ progenitors from healthy individuals and AML patients revealed that it was significantly increased in the cell lines tested as well as in primary patient samples harboring FLT3-ITD, NRAS ${ }^{\mathrm{Q} 1 \mathrm{~L}}$, and JAK3 ${ }^{\mathrm{A572V}}$ genetic lesions (54).

In AML, the coexistence of PP2A-pathway abnormalities with well-known unfavorable molecular markers may provide novel explanations for the worse prognosis associated with those cases.

\section{ACUTE LYMPHOBLASTIC LEUKEMIA}

Almost $20 \%$ of adult patients affected by B-cell ALL harbor the Philadelphia chromosome translocation Ph1, t(9;22)(q34;q11), which usually differs from CML cases because the $B C R / A B L 1$ gene product has a molecular weight of $190 \mathrm{kDa}$ (p190) (55). Although the inclusion of TKIs in the treatment of $P h 1^{+} A L L$ has significantly improved the proportion of patient achieving complete remission, it remains an incurable disease with a very dismal prognosis (56). In Ph1 ALL progenitors, the inhibition of PP2A activity occurs in a SET-dependent manner and the reactivation of the PP2A by FTY720 causes growth inhibition and induction of caspase-dependent apoptosis toward p190 down-regulation (38). Interestingly, the induction of apoptosis and inhibition of cytokine-dependent clonogenic potential are observed regardless of the presence of T315I mutation and without compromising normal myelopoiesis, stressing the concept that FTY720 could represent a feasible and effective treatment also in highly resistant Ph1+ ALL (and CML) cases (38).

\section{T-cell ALL}

$T$-cell $A L L(T-A L L)$ is a malignancy often characterized by aggressive clinical outcome, high relapse risk, and poor prognosis (57). Recurrent chromosomal aberrations and gene mutations have been identified, although a consistent proportion of patients lack molecular or cytogenetic abnormalities (58). By combining arrayCGH (comparative genomic hybridization) and gene expression profiling (GEP), a recurrent deletion at $9 \mathrm{q} 34$ was identified among patients with T-ALL (59). The $\operatorname{del}(9)(\mathrm{q} 34.11 \mathrm{q} 34.13)$ results in SET-NUP214 fusion gene that binds in the promoter regions of the HOXA gene cluster leading to transcriptional activation of HOXA9 and HOXA10 probably via CRM1 and DOT1L. In this study, the knock-down of SET-NUP214 by siRNA down regulated the HOXA expression levels with only mild effect on cell proliferation and apoptosis. Although conceptually SET-NUP214 fusion protein might affect PP2A activity, no evidence has been revealed in this regard.

Although the paucity of data regarding the involvement of PP2A or SET in ALL could not draw any conclusive remark, the presence of the Ph1 chromosome in a cohort of patients with ALL would allow in the next future to investigate the role of PADs in this subgroup of patients and to test whether the inclusion of PADs in 
standard treatment schedule may add any advantage in cure rate and survivals.

\section{B-CHRONIC LYMPHOCYTIC LEUKEMIA AND NON-HODGKIN LYMPHOMAS}

B-chronic lymphocytic leukemia (B-CLL) is the most common leukemia in the Western world, and is characterized by a highly heterogeneous clinical course (60). In a selected group of patients with CLL harboring deletion 11q-, reduced PP2A-A $\beta$ subunit (PPP2R1B) transcript levels were observed. Likewise, alternative splicing of $\mathrm{PP} 2 \mathrm{~A}-\mathrm{A} \beta$ transcripts was associated with a reduced activity of PP2A (61). To further confirmation that PP2A signaling is involved in lymphoid malignancies, SET was found overexpressed in primary CLL cells and B-cell non-Hodgkin lymphoma (NHL) cell line cells in comparison with normal B-cells (62). In patients with CLL, elevated SET levels associated differently with known prognostic factors, e.g., Rai stage, CD38 expression, and IGVH mutational status. Moreover, in CLL cells, increased levels of SET correlated significantly with disease severity (shorter time to treatment and overall survival) (62).

The crucial role of PP2A tumor suppressor in NHL is confirmed by the promising results that FTY720 has shown in pre-clinical experiments in mantle cell lymphoma (MCL), an aggressive Bcell malignancy characterized by $\mathrm{t}(11 ; 14)$ translocation and cyclin D1 expression $(39,63)$. In MCL cell lines, the FTY720-induced cytotoxicity occurs independent of caspase activation and is associated with downmodulation of cyclin D1 $(39,63)$, phospho-Akt (39), and the formation of reactive oxygen species (ROS) (63) and lysosomal membrane permeabilization (39).

The demonstration that the PP2A pathway is affected either in myeloid or in lymphoid chronic and acute malignancies underscores the importance of this tumor suppressor and widens the spectrum of malignancies that may potentially be treated by PADs.

\section{PP2A AND miRNA}

MiRNAs are small (20-24 nucleotide) non-coding RNAs that target messenger RNA for degradation or translational repression and also bind RNA binding protein to control their activity on mRNAs (64-66). Similarly to coding gene, miRNAs could act as oncogene or tumor suppressor depending on how their expression levels associate with cancer (67). Although the contribution of miRNAs to the leukemic transformation or progression has been clearly demonstrated by different groups, few studies have focused on the possible interplay between PP2A pathway and miRNAs in hematological malignancies. Mavrakis and collaborators found that miR-19 is highly expressed in ALL and in aggressive B-cell lymphoma; in a single patient with T-ALL, they identified two novel translocations, $\mathrm{t}(9 ; 14)(\mathrm{q} 34 ; \mathrm{q} 11)$ and $\mathrm{t}(13 ; 14)(\mathrm{q} 32 ; \mathrm{q} 11)$, involving simultaneously Notch1 and the 17-92 cluster including miR-19 (68). Using an unbiased shRNA screening approach, they found eight genes whose knock-down resembles miR-19 effects in vitro, including BIM and PP2A (Ppp2r5e). Hence, in Notchinduced T-ALL miR-19 overexpression may repress PP2A and BIM, thus impairing cell survival and apoptosis $(68,69)$.

Except for miR-19, only a few data have provided evidence of the possible role of miRNAs in regulating PP2A activity and mostly relate to miRNA targeting specific PP2A B subunits (70). MiRNAs might inhibit PP2A transcription (similarly to miR-19) or regulate the expression of PP2A inhibitory molecules (e.g., CIP2A and SET) (71). Moreover, miRNAs can indirectly affect the PP2A pathway by altering the expression of many proteins that cross-talk with PP2A.

\section{PP2A-ACTIVATING DRUGS}

Genetic (SET shRNA-mediated down-regulation) or pharmacologic restoration (i.e., PADs) of PP2A activity halts malignant cell survival and proliferation both in vitro and in different animal models of leukemia.

The first drugs to be tested were forskolin, a diterpene derived from the roots of Coleus forskohlii, and its derivative 1,9dideoxyforskolin, which lacks the adenylate cyclase activity but retains the ability to inhibit PP2A (44) (Table 2). In CML and in $\mathrm{Ph}^{+} \mathrm{ALL}$, forskolin may suppress BCR/ABL1 oncogenic potential in vitro. Interestingly, the anti-leukemic effects of forskolin was observed either in IM-sensitive or in -resistant BCR/ABL1 ${ }^{+}$cell lines including cell lines harboring the T315I mutation that confers resistance to most TKIs (44). Furthermore, PP2A-activation results in growth suppression, enhanced apoptosis, restored differentiation, impaired clonogenic potential, and decreased leukemogenesis in SCID mice injected with $\mathrm{BCR} / \mathrm{ABL}^{+}$cells regardless of their sensitivity to $\operatorname{IM}(38,44)$. Importantly, forskolin and 1,9-dideoxyforskolin do not affect CD34 ${ }^{+}$normal cells, thus preventing toxic effects on normal hematopoiesis (38).

Although forskolin and 1,9-dideoxyforskolin showed good safety profile and efficacy in pre-clinical studies, the following study-drug phases focused on a new non-immunosuppressive compound, e.g., FTY720 (Fingolimod, Gylenia ${ }^{\mathrm{TM}}$ ), recently approved for the treatment of multiple sclerosis and for prophylaxis of solid organ transplantation rejection $(72,73)$. After phosphorylation by sphingosine kinase 2 (SPHK2), FTY720-P temporary promotes internalization of the sphingosine 1-phosphate receptor (S1PR1). The downregulation of S1PR1 on the surface of lymphocytes prevents their egress from the thymus or lymph-nodes to blood stream. However, S1P/S1PR1 signaling may modulate T-cell trafficking as well as T-helper priming,

\section{Table 2 | PP2A-activating drugs (PADs)}

\begin{tabular}{|c|c|c|c|}
\hline Drugs & $\begin{array}{l}\text { Mechanism of } \\
\text { action }\end{array}$ & $\begin{array}{l}\text { Phase } \\
\text { study }\end{array}$ & $\begin{array}{l}\text { Hematological } \\
\text { malignancies }\end{array}$ \\
\hline $\begin{array}{l}\text { Forskolin/1,9- } \\
\text { dideoxyforskolin }\end{array}$ & PP2A reactivation & 1 & CML (included T315I) \\
\hline Fingolimod & SET inhibitor & I & $\mathrm{CML}, \mathrm{AML}$ \\
\hline OSU-2S & & & $\mathrm{ALL} \mathrm{Ph}^{+}$and $\mathrm{Ph}^{-}$ \\
\hline \multirow[t]{2}{*}{ S-FTY720 } & & & Jak2 ${ }^{\mathrm{V} 617} \mathrm{MPN}$ \\
\hline & & & $\mathrm{B}-\mathrm{CLL}$ and $\mathrm{MCL}$ \\
\hline OP449 & SET inhibitor & I & B-CLL, NHL, CML, AML \\
\hline
\end{tabular}

CML, chronic myeloid leukemia; ALL, acute lymphoid leukemia; Jak2V617MPN, Jak2V617F positive myeloproliferative neoplasia; B-CLL, B-cell chronic lymphocytic leukemia; NHL, non-Hodgkin lymphoma; MCL, mantle cell lymphoma; $A M L$, acute myeloid leukemia. 
probably through the sequestration of T-cells within the antigen encounter sites and the regulation of activation molecule receptors (74). The high oral bioavailability and the absence of harmful side effects prompted the experimentation of FTY720 into clinical phases.

The anti-leukemic effect of FTY720 is thought to rely on the ability to re-activate PP2A and it does not require SPHK2 phosphorylation (75). The S1PR1 agonist (FTY720-P) neither induces PP2A activity nor antagonizes the specific molecular aberrations, for example, BCR-ABL1 or Jak2 pathways $(8,25)$. By contrast, the FTY720 derivatives (e.g., OSU-2S and S-FTY720-regioisomer) do not bind at the docking site of S1PR1 and do not get SPHK2 phosphorylated thereby inactive as immunosuppressive drugs while they still retain the ability to bind SET and activate PP2A $(25,27$, 76). Thus, this second generation FTY720 derivatives will overcome the possible increased conversion to the inactive FTY20-P by lymphoid B and T cells expressing SPHK2 thereby preventing the adverse effects (e.g., lymphopenia or bradycardia), which have been noted in patients with multiple sclerosis at the time of FTY720 therapy initiation (72).

So far, the data of FTY720 efficacy in hematological malignancies are drawn from pre-clinical studies, although the drug has been used in patients with multiple sclerosis. Interestingly, in bone marrow xenografts, PADs nearly abrogated survival of leukemic progenitors and dramatically impaired survival and self-renewal of CML and $\mathrm{Ph} 1^{+} \mathrm{B}-\mathrm{ALL}$ stem and/or progenitor cells, but not of normal quiescent HSCs, through BCR-ABL1independent and PP2A-mediated inhibition of Jak2 and $\beta$-catenin $(27,44)$. This results in apoptosis of $\mathrm{CD}_{3} 4^{+}$progenitors in patients with TKI-sensitive and -resistant CML and translates into long-term survival with normal myelopoiesis and absence of toxic effects in BCR/ABL1-positive leukemic mice (27, 38, 44). These findings could represent a model to selectively target HSCs reservoir sparing normal stem cells. The depletion of leukemic and genetically unstable stem cells has several biological and clinical implications. At first, it would prevent the onset of Ph1 negative sub-clones, which may eventually expand and contribute to disease progression or relapse in all types of $\mathrm{Ph}^{+}$leukemias (77). Second, PADs seem to selectively activate PP2A in leukemic HSCs without harmful events on normal hematopoiesis; this could be the rationale to test the efficacy of PADs in other stem cell-derived malignancies. At last and most importantly, the eradication of leukemic HSCs would theoretically cure patients with CML who may eventually discontinue TKIs treatment.

Recently, Oaks and collaborators provided evidence of FTY720 efficacy in Jak2-driven hematological malignancies (25). PP2A is inactive in $\mathrm{PV}$ and in $\mathrm{Jak} 2^{\mathrm{V} 617 \mathrm{~F}} \mathrm{MPN}$ progenitors, and this occurs in an oncogene dose- and kinase-dependent manner and it is mediated by PP2A inhibitor SET. In Jak2 ${ }^{\mathrm{V} 617 \mathrm{~F}}$ MPN cells, the FTY720 non-immunosuppressive derivatives (OSU-2S and S-FTY720-regioisomer) down-regulate Jak2 through the binding with SET lysine K209, which finally causes the sequestration and inactivation of SET (25). Of interest, in SCID mice injected with BA/F3-Jak2 ${ }^{\mathrm{V} 617 \mathrm{~F}}$ cells, FTY720 long-term treatment is associated with longer survival and decreased leukemic allelic burden without any injury on cardiac performance.
In myeloid and lymphoid cell lines, treatment with FTY720 restores Bcl-2 dephosphorylation and apoptosis $(78,79)$. However, treatment of primary CLL cell lines with FTY720-induced down-regulation of Mcl-1 but not Bcl-2 and activation of ERK1/2 dephosphorylation. These findings may be consistent with the hypothesis that FTY720 apoptosis, in CLL, is Mcl-1 and ERK1/2mediated (80). FTY720 induces apoptosis in a time- and dosedependent manner in primary MCL tumor cells and MCL cell lines in vitro. In addition, in MCL cells FTY720 treatment results in the accumulation of cells in G0-G1 and G2-M phases of the cell cycle. Most importantly, the in vivo therapeutic activity of FTY720 was shown in severe combined immunodeficient mice engrafted with Jeko MCL cell line (63). Interestingly, the rate of cell death is significantly improved in MCL cell lines treated with FTY720 and milatuzumab, a fully humanized monoclonal antibody $(\mathrm{mAb})$ specific for CD74 that increases following induction of autophagic-lysosomal pathway by FTY720 (39).

Pharmacologic activation of PP2A by FTY720, but not FTY720$\mathrm{P}$ (phosphorylated), reduced proliferation, inhibited clonogenic potential, and induced apoptosis in cells harboring the c-KIT mutation, that is frequently encountered in myeloid malignancies, especially AML (40). Similarly, in vivo treatment with FTY720 delayed growth of c-KIT ${ }^{+}$mutant cells as confirmed by shrinkage in spleen size and significantly lower bone marrow infiltration (40).

\section{OP449 (also called COG449)}

OP449 (also called COG449) is a novel, cell penetrating peptide, which acts through the binding with SET, thus preventing the formation of the SET-PP2A complexes (10). Interestingly, OP449 determined a reduction in cellular levels of the antiapoptotic Bcl-2 family member Mcl-1, which is overexpressed in CLL cells (62). In CML and AML cell lines, OP449 in combination with tyrosine kinases and chemotherapy produced efficient and selective inhibition of leukemia cell growth as compared with normal cells, suggesting that SET inhibitors and TKIs or chemotherapy could represent a new drug combination to overcome drug resistance (54). In B-CLL and NHL, OP449 has shown to increase significantly the activity of PP2A and to inhibit growth of tumor xenografts in mice (62).

\section{AAL(S), (2-amino-4-(4-heptyloyphenol)-2-methylbutanol)}

$A A L(S),(2-a m i n o-4-(4-h e p t y l o y p h e n o l)-2-m e t h y l b u t a n o l)$ is a new non-phosphorylatable FTY720 analog that has recently shown to reduce airway hyperreactivity and inflammation in an asthma mouse model (81). In the next future, new FTY720 analogs may be tested in leukemic cells or mouse model to assess their efficacy and tolerability in hematological disorders.

Compounds that are able to target tyrosine kinases along with drugs that act at the epigenetic modifications occurring in leukemic cells are emerging as highly effective in numerous hematological malignancies (82). Sorafenib (a new generation FLT3 mutant inhibitors) and vorinostat (a histone deacetylases inhibitor, HDAC) induce tumor-cell death through the induction of PP2A activity in C16 dihydroceramide-dependent manner (83). Finally, the induction of complete differentiation of leukemic cells through the enhanced expression of PP2A regulatory subunits represents 
a mechanism explaining the efficacy of methylprednisolone in the treatment of lymphoid malignancies (84).

\section{CONCLUSION}

Protein phosphatase $2 \mathrm{~A}$ is a well-characterized phosphatase that has been found suppressed in hematological malignancies. Over the past decades, new evidence of its emerging role as tumor suppressor has been clearly provided. The overexpression or aberrant expression of PP2A inhibitory molecules, like SET or SETBP1 and CIP2A, contributes to PP2A loss-of-function. In other cases, PP2A activity is suppressed by unrestrained oncogene signaling driven by aberrant kinase activity (e.g., BCR-ABL1, Jak2 ${ }^{\mathrm{V} 617 \mathrm{~F}}$ ). Numerous studies have shown that PP2A loss-of-function predict shorter survival independently from other prognostic factors in myeloid and lymphoid malignancies, and disease progression/TKI response in CML. An interesting finding that has emerged recently is that suppression of PP2A is crucial for the persistence of leukemic HSCs in CML. Hence, PP2A re-activating drugs might enable to selectively target the leukemic HSCs that show innate resistance to TKIs allowing the eradication of the leukemic reservoir and the risk of disease relapse or progression. FTY720 and its non-immunosuppressive derivatives offered promising results in pre-clinical models. Although further studies are warranted to better understand how PP2A inhibition influences leukemia emergence, maintenance, and/or progression, the data reviewed herein strongly indicate that clinical trials are now imperative to evaluate the efficacy of PADs in the treatment of patients with hematologic malignancies.

\section{AUTHOR CONTRIBUTIONS}

Maria Ciccone, George A. Calin, and Danilo Perrotti wrote the manuscript.

\section{ACKNOWLEDGMENTS}

Dr. Calin is The Alan M. Gewirtz Leukemia and Lymphoma Society Scholar. Work in Dr. Calin's laboratory is supported in part by the NIH/NCI grants 1UH2TR00943-01 and 1 R01 CA182905-01, the Department of Defense, a Developmental Research Award in Leukemia SPORE, a SINF MDACC-DKFZ grant in CLL, the Duncan Family Institutional Seed Funds, The Blanton-Davis Ovarian Cancer - 2013 Sprint for Life Research Award, the Laura and John Arnold Foundation, the RGK Foundation, and the Estate of C. G. Johnson, Jr. This work was supported in part by the NIH-NCI CA163800 (Danilo Perrotti). Dr. Ciccone is partially supported by A. I. L. - Associazione Italiana contro le Leucemia, Linfomi e Mielomi - Sezione di Ferrara.

\section{REFERENCES}

1. Bononi A, Agnoletto C, De Marchi E, Marchi S, Patergnani S, Bonora M, et al. Protein kinases and phosphatases in the control of cell fate. Enzyme Res (2011) 2011:329098. doi:10.4061/2011/329098

2. Rowley JD. Letter: a new consistent chromosomal abnormality in chronic myelogenous leukaemia identified by quinacrine fluorescence and Giemsa staining. Nature (1973) 243(5405):290-3. doi:10.1038/243290a0

3. Westermarck J, Hahn WC. Multiple pathways regulated by the tumor suppressor PP2A in transformation. Trends Mol Med (2008) 14(4):152-60. doi:10.1016/j. molmed.2008.02.001

4. Perrotti D, Jamieson C, Goldman J, Skorski T. Chronic myeloid leukemia: mechanisms of blastic transformation. J Clin Invest (2010) 120(7):2254-64. doi:10.1172/JCI41246
5. Druker BJ, Talpaz M, Resta DJ, Peng B, Buchdunger E, Ford JM, et al. Efficacy and safety of a specific inhibitor of the BCR-ABL tyrosine kinase in chronic myeloid leukemia. N Engl J Med (2001) 344(14):1031-7. doi:10.1056/ NEJM200104053441401

6. Eifert C, Powers RS. From cancer genomes to oncogenic drivers, tumour dependencies and therapeutic targets. Nat Rev Cancer (2012) 12(8):572-8. doi: $10.1038 / \mathrm{nrc} 3299$

7. Millward TA, Zolnierowicz S, Hemmings BA. Regulation of protein kinase cascades by protein phosphatase 2A. Trends Biochem Sci (1999) 24(5):186-91. doi:10.1016/S0968-0004(99)01375-4

8. Perrotti D, Neviani P. Protein phosphatase 2A: a target for anticancer therapy. Lancet Oncol (2013) 14(6):e229-38. doi:10.1016/S1470-2045(12)70558-2

9. Calin GA, di Iasio MG, Caprini E, Vorechovsky I, Natali PG, Sozzi G, et al. Low frequency of alterations of the alpha (PPP2R1A) and beta (PPP2R1B) isoforms of the subunit A of the serine-threonine phosphatase $2 \mathrm{~A}$ in human neoplasms. Oncogene (2000) 19(9):1191-5. doi:10.1038/sj.onc.1203389

10. Neviani P, Perrotti D. SETting OP449 into the PP2A-activating drug family. Clin Cancer Res (2014) 20(8):2026-8. doi:10.1158/1078-0432.CCR-14-0166

11. Cohen P. The structure and regulation of protein phosphatases. Annu Rev Biochem (1989) 58:453-508. doi:10.1146/annurev.bi.58.070189.002321

12. Eichhorn PJ, Creyghton MP, Bernards R. Protein phosphatase $2 \mathrm{~A}$ regulatory subunits and cancer. Biochim Biophys Acta (2009) 1795(1):1-15. doi:10.1016/j. bbcan.2008.05.005

13. Cegielska A, Shaffer S, Derua R, Goris J, Virshup DM. Different oligomeric forms of protein phosphatase $2 \mathrm{~A}$ activate and inhibit simian virus 40 DNA replication. Mol Cell Biol (1994) 14(7):4616-23.

14. Everett AD, Kamibayashi C, Brautigan DL. Transgenic expression of protein phosphatase 2A regulatory subunit B56gamma disrupts distal lung differentiation. Am J Physiol Lung Cell Mol Physiol (2002) 282(6):L1266-71. doi:10.1152/ajplung.00262.2001

15. Strack S. Overexpression of the protein phosphatase $2 \mathrm{~A}$ regulatory subunit Bgamma promotes neuronal differentiation by activating the MAP kinase (MAPK) cascade. J Biol Chem (2002) 277(44):41525-32. doi:10.1074/jbc. M203767200

16. Polakis P. Wnt signaling and cancer. Genes Dev (2000) 14(15):1837-51. doi:10. 1101/gad.14.15.1837

17. Kurimchak A, Grana X. PP2A holoenzymes negatively and positively regulate cell cycle progression by dephosphorylating pocket proteins and multiple CDK substrates. Gene (2012) 499(1):1-7. doi:10.1016/j.gene.2012.02.015

18. Perrotti D, Neviani P. Protein phosphatase 2A (PP2A), a druggable tumor suppressor in Ph1(+) leukemias. Cancer Metastasis Rev (2008) 27(2):159-68. doi:10.1007/s10555-008-9119-x

19. McCourt P, Gallo-Ebert C, Gonghong Y, Jiang Y, Nickels JT Jr. PP2A(Cdc55) regulates G1 cyclin stability. Cell Cycle (2013) 12(8):1201-10. doi:10.4161/cc.24231

20. Ma J, Arnold HK, Lilly MB, Sears RC, Kraft AS. Negative regulation of Pim1 protein kinase levels by the B56beta subunit of PP2A. Oncogene (2007) 26(35):5145-53. doi:10.1038/sj.onc.1210323

21. Aho TL, Sandholm J, Peltola KJ, Mankonen HP, Lilly M, Koskinen PJ. Pim-1 kinase promotes inactivation of the pro-apoptotic Bad protein by phosphorylating it on the Ser112 gatekeeper site. FEBS Lett (2004) 571(1-3):43-9. doi:10.1016/j.febslet.2004.06.050

22. Andrabi S, Gjoerup OV, Kean JA, Roberts TM, Schaffhausen B. Protein phosphatase $2 \mathrm{~A}$ regulates life and death decisions via Akt in a context-dependent manner. Proc Natl Acad Sci USA (2007) 104(48):19011-6. doi:10.1073/pnas. 0706696104

23. Janssens V, Longin S, Goris J. PP2A holoenzyme assembly: in cauda venenum (the sting is in the tail). Trends Biochem Sci (2008) 33(3):113-21. doi:10.1016/j. tibs.2007.12.004

24. Samanta AK, Chakraborty SN, Wang Y, Kantarjian H, Sun X, Hood J, et al. Jak2 inhibition deactivates Lyn kinase through the SET-PP2A-SHP1 pathway, causing apoptosis in drug-resistant cells from chronic myelogenous leukemia patients. Oncogene (2009) 28(14):1669-81. doi:10.1038/onc.2009.7

25. Oaks JJ, Santhanam R, Walker CJ, Roof S, Harb JG, Ferenchak G, et al. Antagonistic activities of the immunomodulator and PP2A-activating drug FTY720 (Fingolimod, Gilenya) in Jak2-driven hematologic malignancies. Blood (2013) 122(11):1923-34. doi:10.1182/blood-2013-03-492181

26. Yokoyama N, Reich NC, Miller WT. Involvement of protein phosphatase $2 \mathrm{~A}$ in the interleukin-3-stimulated Jak2-Stat5 signaling pathway. J Interferon Cytokine Res (2001) 21(6):369-78. doi:10.1089/107999001750277844 
27. Neviani P, Harb JG, Oaks JJ, Santhanam R, Walker CJ, Ellis JJ, et al. PP2Aactivating drugs selectively eradicate TKI-resistant chronic myeloid leukemic stem cells. J Clin Invest (2013) 123(10):4144-57. doi:10.1172/JCI68951

28. Cristobal I, Blanco FJ, Garcia-Orti L, Marcotegui N, Vicente C, Rifon J, et al. SETBP1 overexpression is a novel leukemogenic mechanism that predicts adverse outcome in elderly patients with acute myeloid leukemia. Blood (2010) 115(3):615-25. doi:10.1182/blood-2009-06-227363

29. Beresford PJ, Zhang D, Oh DY, Fan Z, Greer EL, Russo ML, et al. Granzyme A activates an endoplasmic reticulum-associated caspase-independent nuclease to induce single-stranded DNA nicks. J Biol Chem (2001) 276(46):43285-93. doi:10.1074/jbc.M108137200

30. Fan Z, Beresford PJ, Oh DY, Zhang D, Lieberman J. Tumor suppressor NM23$\mathrm{H} 1$ is a granzyme A-activated DNase during CTL-mediated apoptosis, and the nucleosome assembly protein SET is its inhibitor. Cell (2003) 112(5):659-72. doi:10.1016/S0092-8674(03)00150-8

31. Minakuchi M, Kakazu N, Gorrin-Rivas MJ, Abe T, Copeland TD, Ueda K, et al. Identification and characterization of SEB, a novel protein that binds to the acute undifferentiated leukemia-associated protein SET. Eur J Biochem (2001) 268(5):1340-51. doi:10.1046/j.1432-1327.2001.02000.x

32. Hoischen A, van Bon BW, Gilissen C, Arts P, van Lier B, Steehouwer M, et al. De novo mutations of SETBP1 cause Schinzel-Giedion syndrome. Nat Genet (2010) 42(6):483-5. doi:10.1038/ng.581

33. Meggendorfer M, Bacher U, Alpermann T, Haferlach C, Kern W, GambacortiPasserini C, et al. SETBP1 mutations occur in $9 \%$ of MDS/MPN and in $4 \%$ of MPN cases and are strongly associated with atypical CML, monosomy 7, isochromosome $\mathrm{i}(17)(\mathrm{q} 10)$, ASXL1 and CBL mutations. Leukemia (2013) 27(9):1852-60. doi:10.1038/leu.2013.133

34. Piazza R, Valletta S, Winkelmann N, Redaelli S, Spinelli R, Pirola A, et al. Recurrent SETBP1 mutations in atypical chronic myeloid leukemia. Nat Genet (2013) 45(1):18-24. doi:10.1038/ng.2495

35. Junttila MR, Puustinen P, Niemela M, Ahola R, Arnold H, Bottzauw T, et al. CIP2A inhibits PP2A in human malignancies. Cell (2007) 130(1):51-62. doi:10.1016/j.cell.2007.04.044

36. Laine A, Sihto H, Come C, Rosenfeldt MT, Zwolinska A, Niemela M, et al. Senescence sensitivity of breast cancer cells is defined by positive feedback loop between CIP2A and E2F1. Cancer Discov (2013) 3(2):182-97. doi:10.1158/21598290.CD-12-0292

37. Jin L, Liu G, Zhang CH, Lu CH, Xiong S, Zhang MY, et al. Nm23-H1 regulates the proliferation and differentiation of the human chronic myeloid leukemia K562 cell line: a functional proteomics study. Life Sci (2009) 84(13-14):458-67. doi:10.1016/j.lfs.2009.01.010

38. Neviani P, Santhanam R, Oaks JJ, Eiring AM, Notari M, Blaser BW, et al. FTY720, a new alternative for treating blast crisis chronic myelogenous leukemia and Philadelphia chromosome-positive acute lymphocytic leukemia. J Clin Invest (2007) 117(9):2408-21. doi:10.1172/JCI31095

39. Alinari L, Mahoney E, Patton J, Zhang X, Huynh L, Earl CT, et al. FTY720 increases CD74 expression and sensitizes mantle cell lymphoma cells to milatuzumab-mediated cell death. Blood (2011) 118(26):6893-903. doi:10. 1182/blood-2011-06-363879

40. Roberts KG, Smith AM, McDougall F, Carpenter H, Horan M, Neviani P, et al. Essential requirement for PP2A inhibition by the oncogenic receptor c-KIT suggests PP2A reactivation as a strategy to treat c-KIT+ cancers. Cancer Res (2010) 70(13):5438-47. doi:10.1158/0008-5472.CAN-09-2544

41. Hochhaus A, O’Brien SG, Guilhot F, Druker BJ, Branford S, Foroni L, et al. Six-year follow-up of patients receiving imatinib for the first-line treatment of chronic myeloid leukemia. Leukemia (2009) 23(6):1054-61. doi:10.1038/leu. 2009.38

42. Soverini S, Colarossi S, Gnani A, Rosti G, Castagnetti F, Poerio A, et al. Contribution of $\mathrm{ABL}$ kinase domain mutations to imatinib resistance in different subsets of Philadelphia-positive patients: by the GIMEMA working party on chronic myeloid leukemia. Clin Cancer Res (2006) 12(24):7374-9. doi:10.1158/1078-0432.CCR-06-1516

43. Pellicano F, Sinclair A, Holyoake TL. In search of CML stem cells' deadly weakness. Curr Hematol Malig Rep (2011) 6(2):82-7. doi:10.1007/s11899-0110085-y

44. Neviani P, Santhanam R, Trotta R, Notari M, Blaser BW, Liu S, et al. The tumor suppressor PP2A is functionally inactivated in blast crisis CML through the inhibitory activity of the BCR/ABL-regulated SET protein. Cancer Cell (2005) 8(5):355-68. doi:10.1016/j.ccr.2005.10.015
45. Salas A, Ponnusamy S, Senkal CE, Meyers-Needham M, Selvam SP, Saddoughi SA, et al. Sphingosine kinase-1 and sphingosine 1-phosphate receptor 2 mediate Bcr-Abll stability and drug resistance by modulation of protein phosphatase 2A. Blood (2011) 117(22):5941-52. doi:10.1182/blood-2010-08-300772

46. Khanna A, Bockelman C, Hemmes A, Junttila MR, Wiksten JP, Lundin M, et al. MYC-dependent regulation and prognostic role of CIP2A in gastric cancer. $J$ Natl Cancer Inst (2009) 101(11):793-805. doi:10.1093/jnci/djp103

47. Wang J, Li W, Li L, Yu X, Jia J, Chen C. CIP2A is over-expressed in acute myeloid leukaemia and associated with HL60 cells proliferation and differentiation. Int J Lab Hematol (2011) 33(3):290-8. doi:10.1111/j.1751-553X.2010.01288.x

48. Lucas CM, Harris RJ, Giannoudis A, Copland M, Slupsky JR, Clark RE. Cancerous inhibitor of PP2A (CIP2A) at diagnosis of chronic myeloid leukemia is a critical determinant of disease progression. Blood (2011) 117(24):6660-8. doi:10.1182/blood-2010-08-304477

49. Makishima H, Yoshida K, Nguyen N, Przychodzen B, Sanada M, Okuno Y, et al. Somatic SETBP1 mutations in myeloid malignancies. Nat Genet (2013) 45(8):942-6. doi:10.1038/ng.2696

50. Hou HA, Kuo YY, Tang JL, Chou WC, Yao M, Lai YJ, et al. Clinical implications of the SETBP1 mutation in patients with primary myelodysplastic syndrome and its stability during disease progression. Am J Hematol (2014) 89(2):181-6. doi:10.1002/ajh.23611

51. Linnekin D. Early signaling pathways activated by c-Kit in hematopoietic cells. Int J Biochem Cell Biol (1999) 31(10):1053-74. doi:10.1016/S1357-2725(99) 00078-3

52. Mrozek K, Marcucci G, Paschka P, Bloomfield CD. Advances in molecular genetics and treatment of core-binding factor acute myeloid leukemia. Curr Opin Oncol (2008) 20(6):711-8. doi:10.1097/CCO.0b013e32831369df

53. Cristobal I, Garcia-Orti L, Cirauqui C, Alonso MM, Calasanz MJ, Odero MD. PP2A impaired activity is a common event in acute myeloid leukemia and its activation by forskolin has a potent anti-leukemic effect. Leukemia (2011) 25(4):606-14. doi:10.1038/leu.2010.294

54. Agarwal A, Mackenzie RJ, Pippa R, Eide CA, Oddo J, Tyner JW, et al. Antagonism of SET using OP449 enhances the efficacy of tyrosine kinase inhibitors and overcomes drug resistance in myeloid leukemia. Clin Cancer Res (2014) 20(8):2092-103. doi:10.1158/1078-0432.CCR-13-2575

55. Westbrook CA, Hooberman AL, Spino C, Dodge RK, Larson RA, Davey F, et al. Clinical significance of the BCR-ABL fusion gene in adult acute lymphoblastic leukemia: a Cancer and leukemia group B study (8762). Blood (1992) 80(12):2983-90.

56. Fielding AK. Current treatment of Philadelphia chromosome-positive acute lymphoblastic leukemia. Hematology Am Soc Hematol Educ Program (2011) 2011:231-7. doi:10.1182/asheducation-2011.1.231

57. Pui CH, Relling MV, Downing JR. Acute lymphoblastic leukemia. N Engl J Med (2004) 350(15):1535-48. doi:10.1056/NEJMra023001

58. van Grotel M, Meijerink JP, Beverloo HB, Langerak AW, Buys-Gladdines JG, Schneider $\mathrm{P}$, et al. The outcome of molecular-cytogenetic subgroups in pediatric T-cell acute lymphoblastic leukemia: a retrospective study of patients treated according to DCOG or COALL protocols. Haematologica (2006) 91(9): 1212-21.

59. Van Vlierberghe P, van Grotel M, Tchinda J, Lee C, Beverloo HB, van der Spek PJ, et al. The recurrent SET-NUP214 fusion as a new HOXA activation mechanism in pediatric T-cell acute lymphoblastic leukemia. Blood (2008) 111(9):4668-80. doi:10.1182/blood-2007-09-111872

60. Chiorazzi N, Rai KR, Ferrarini M. Chronic lymphocytic leukemia. N Engl J Med (2005) 352(8):804-15. doi:10.1056/NEJMra041720

61. Kalla C, Scheuermann MO, Kube I, Schlotter M, Mertens D, Dohner H, et al. Analysis of 11q22-q23 deletion target genes in B-cell chronic lymphocytic leukaemia: evidence for a pathogenic role of NPAT, CUL5, and PPP2R1B. Eur J Cancer (2007) 43(8):1328-35. doi:10.1016/j.ejca.2007.02.005

62. Christensen DJ, Chen Y, Oddo J, Matta KM, Neil J, Davis ED, et al. SET oncoprotein overexpression in B-cell chronic lymphocytic leukemia and non-Hodgkin lymphoma: a predictor of aggressive disease and a new treatment target. Blood (2011) 118(15):4150-8. doi:10.1182/blood-2011-04-351072

63. Liu Q, Alinari L, Chen CS, Yan F, Dalton JT, Lapalombella R, et al. FTY720 shows promising in vitro and in vivo preclinical activity by downmodulating Cyclin D1 and phospho-Akt in mantle cell lymphoma. Clin Cancer Res (2010) 16(12):3182-92. doi:10.1158/1078-0432.CCR-09-2484

64. Spizzo R, Nicoloso MS, Croce CM, Calin GA. SnapShot: microRNAs in cancer. Cell (2009) 137(3):586-el. doi:10.1016/j.cell.2009.04.040 
65. Eiring AM, Harb JG, Neviani P, Garton C, Oaks JJ, Spizzo R, et al. miR-328 functions as an RNA decoy to modulate hnRNP E2 regulation of mRNA translation in leukemic blasts. Cell (2010) 140(5):652-65. doi:10.1016/j.cell.2010.01.007

66. Balkhi MY, Iwenofu OH, Bakkar N, Ladner KJ, Chandler DS, Houghton PJ, et al. miR-29 acts as a decoy in sarcomas to protect the tumor suppressor A20 mRNA from degradation by HuR. Sci Signal (2013) 6(286):ra63. doi:10.1126/scisignal.2004177

67. Calin GA, Croce CM. Chronic lymphocytic leukemia: interplay between noncoding RNAs and protein-coding genes. Blood (2009) 114(23):4761-70. doi:10. 1182/blood-2009-07-192740

68. Mavrakis KJ, Wolfe AL, Oricchio E, Palomero T, de Keersmaecker K, McJunkin K, et al. Genome-wide RNA-mediated interference screen identifies miR-19 targets in Notch-induced T-cell acute lymphoblastic leukaemia. Nat Cell Biol (2010) 12(4):372-9. doi: $10.1038 /$ ncb2037

69. Hales EC, Taub JW, Matherly LH. New insights into Notch1 regulation of the PI3K-AKT-mTOR1 signaling axis: targeted therapy of gamma-secretase inhibitor resistant T-cell acute lymphoblastic leukemia. Cell Signal (2014) 26(1):149-61. doi:10.1016/j.cellsig.2013.09.021

70. Liu X, Sempere LF, Ouyang H, Memoli VA, Andrew AS, Luo Y, et al. MicroRNA31 functions as an oncogenic microRNA in mouse and human lung cancer cells by repressing specific tumor suppressors. J Clin Invest (2010) 120(4):1298-309. doi:10.1172/JCI39566

71. Jung HM, Patel RS, Phillips BL, Wang H, Cohen DM, Reinhold WC, et al. Tumor suppressor miR-375 regulates MYC expression via repression of CIP2A coding sequence through multiple miRNA-mRNA interactions. Mol Biol Cell (2013) 24(11):1638-48. doi:10.1091/mbc.E12-12-0891 S1-7,

72. Kappos L, Radue EW, O’Connor P, Polman C, Hohlfeld R, Calabresi P, et al. A placebo-controlled trial of oral fingolimod in relapsing multiple sclerosis. $N$ Engl J Med (2010) 362(5):387-401. doi:10.1056/NEJMoa0909494

73. Kovarik JM, Tedesco-Silva H, Lorber MI, Foster C. Exposure-efficacy relationships of a fingolimod-everolimus regimen in kidney transplant patients at risk for delayed graft function. Transplant Proc (2006) 38(10):3479-82. doi:10.1016/j.transproceed.2006.10.084

74. Garris CS, Blaho VA, Hla T, Han MH. Sphingosine-1-phosphate receptor 1 (S1P) signaling in T cells: trafficking and beyond. Immunology (2014) 142(3):347-53. doi:10.1111/imm.12272

75. Ingwersen J, Aktas O, Kuery P, Kieseier B, Boyko A, Hartung HP. Fingolimod in multiple sclerosis: mechanisms of action and clinical efficacy. Clin Immunol (2012) 142(1):15-24. doi:10.1016/j.clim.2011.05.005

76. Saddoughi SA, Gencer S, Peterson YK, Ward KE, Mukhopadhyay A, Oaks J, et al. Sphingosine analogue drug FTY720 targets I2PP2A/SET and mediates lung tumour suppression via activation of PP2A-RIPK1-dependent necroptosis. EMBO Mol Med (2013) 5(1):105-21. doi:10.1002/emmm.201201283

77. Rizzotto L, Saccenti E, Sofritti O, Daghia G, Volta E, Caprini E, et al. BCR/ABL1positive acute lymphoblastic leukemia relapsing as BCR/ABL1-negative acute lymphoblastic leukemia. Leuk Lymphoma (2013) 54(9):2065-7. doi:10.3109/ 10428194.2012.762513

78. Cheng X, Bennett RL, Liu X, Byrne M, Stratford May W. PKR negatively regulates leukemia progression in association with PP2A activation, Bcl-2 inhibition and increased apoptosis. Blood Cancer J (2013) 3:e144. doi:10.1038/bcj.2013.42

79. Deng X, Gao F, May WS. Protein phosphatase 2A inactivates Bcl2's antiapoptotic function by dephosphorylation and up-regulation of Bcl2-p53 binding. Blood (2009) 113(2):422-8. doi:10.1182/blood-2008-06-165134

80. Liu Q, Zhao X, Frissora F, Ma Y, Santhanam R, Jarjoura D, et al. FTY720 demonstrates promising preclinical activity for chronic lymphocytic leukemia and lymphoblastic leukemia/lymphoma. Blood (2008) 111(1):275-84. doi:10.1182/ blood-2006-10-053884

81. Collison A, Hatchwell L, Verrills N, Wark PA, de Siqueira AP, Tooze M, et al. The E3 ubiquitin ligase midline 1 promotes allergen and rhinovirus-induced asthma by inhibiting protein phosphatase 2A activity. Nat Med (2013) 19(2):232-7. doi: $10.1038 / \mathrm{nm} .3049$

82. Galm O, Herman JG, Baylin SB. The fundamental role of epigenetics in hematopoietic malignancies. Blood Rev (2006) 20(1):1-13. doi:10.1016/j.blre. 2005.01.006

83. Aad G, Abbott B, Abdallah J, Abdelalim AA, Abdesselam A, Abdinov O, et al Search for new particles in two-jet final states in $7 \mathrm{TeV}$ proton-proton collisions with the ATLAS detector at the LHC. Phys Rev Lett (2010) 105(16):161801. doi:10.1103/PhysRevLett.105.161801

84. Yuksel S, Saydam G, Uslu R, Sanli UA, Terzioglu E, Buyukececi F, et al. Arsenic trioxide and methylprednisolone use different signal transduction pathways in leukemic differentiation. Leuk Res (2002) 26(4):391-8. doi:10.1016/S01452126(01)00147-3

Conflict of Interest Statement: The authors declare that the research was conducted in the absence of any commercial or financial relationships that could be construed as a potential conflict of interest.

Received: 28 May 2014; accepted: 16 January 2015; published online: 16 February 2015. Citation: Ciccone M, Calin GA and Perrotti D (2015) From the biology of PP2A to the PADs for therapy of hematologic malignancies. Front. Oncol. 5:21. doi: 10.3389/fonc. 2015.00021

This article was submitted to Hematology Oncology, a section of the journal Frontiers in Oncology.

Copyright (c) 2015 Ciccone, Calin and Perrotti. This is an open-access article distributed under the terms of the Creative Commons Attribution License (CC BY). The use, distribution or reproduction in other forums is permitted, provided the original author(s) or licensor are credited and that the original publication in this journal is cited, in accordance with accepted academic practice. No use, distribution or reproduction is permitted which does not comply with these terms. 